\title{
Valoración de las Competencias Investigativas de los Estudiantes de Posgrado en Administración
}

\author{
Edgar O. Cardoso ${ }^{1 *}$ y María T. Cerecedo ${ }^{1}$ \\ (1) Instituto Politécnico Nacional (IPN), Escuela Superior de Comercio y Administración (ESCA), Unidad \\ Santo Tomás, Sección de Estudios de Posgrado e Investigación, Ciudad de México, México \\ (e-mail: eoce@hotmail.com, tricermer@yahoo.com)
}

* Autor a quien debe ser dirigida la correspondencia

Recibido Abr. 6, 2018; Aceptado May. 29, 2018; Versión final Jun. 25, 2018, Publicado Feb. 2019

\begin{abstract}
Resumen
El objetivo principal del estudio fue valorar los niveles de desarrollo de las competencias investigativas del estudiantado en los posgrados de administración en una universidad mexicana. El método que se empleó fue con un enfoque cuantitativo de tipo exploratorio-descriptivo. El instrumento para obtener la información de campo fue un cuestionario basado en una escala Likert. Los ítems se midieron a través de una escala con cinco opciones desde 0 (no desarrollada) hasta 4 (muy desarrollada). La muestra fue de tipo estratificado porque estuvo constituida por 150 alumnos de seis programas de posgrado en administración con una distribución de $73 \%$ de mujeres y $27 \%$ de hombres. Los principales resultados fueron que el estudiantado posee un nivel de desarrollo insuficiente en sus competencias investigativas sobre diseño, instrumentales y gestión de la divulgación del conocimiento. La relevancia del estudio fue brindar información sobre los perfiles de ingreso del estudiantado que accede a una formación de nivel posgrado.
\end{abstract}

\section{Assessment of the Research Competences of Students in Graduate Courses in Administration}

\begin{abstract}
The main objective of the study was to assess the levels of development of the research competences of the students in the graduate courses of administration in a Mexican university. The method that was used was with a quantitative approach of exploratory-descriptive type. The instrument to obtain the field information was a questionnaire based on a Likert scale. The items were measured through a scale with five options from 0 (not developed) to 4 (highly developed). The sample was stratified because it was made up of 150 students from six graduate programs in administration with a distribution of $73 \%$ women and $27 \%$ men. The main results were that students have an insufficient level of development in its research skills on design, instrumental and management of knowledge dissemination. The relevance of the study was to provide information on the entrance profiles of students who access graduate education.
\end{abstract}

Keywords: research competences; assessment; postgraduate; student; administration 


\section{INTRODUCCIÓN}

En la actualidad, la educación superior se ha convertido en el factor relevante que permite conseguir el desarrollo de los países a partir de elevar la competitividad de sus economías (Akareem y Hossain, 2012 y Brennan y Teichler, 2008). Para lograrlo, las instituciones de educación superior (IES) han reorientado sus funciones hacia la generación de conocimiento en vinculación con las empresas grandes tanto privadas como públicas; apoyan el desarrollo de empresas medianas y pequeñas de carácter regional; realizan la formación de fuerza de trabajo considerando competencias basadas en la investigación e innovación, así como ofrecen capital humano calificado para el sector local del país (Carneiro y Draxler, 2008). Además: "Las IES desempeñan un papel fundamental en la formación de profesionales, especialistas de alto nivel, científicos e investigadores que el país demanda. En este contexto, una prioridad es asegurar que estén funcionando en la vanguardia del desarrollo intelectual y científico" (Mercado, Cernas y Nava, 2016:61-62).

En este sentido, Delamare y Winterton (2007) establecieron que la educación superior, especialmente el posgrado ofreciera una formación profesional acorde con los avances científicos y tecnológicos del sector laboral, los cuales en el marco de la globalización económica han cambiado no solo en el ámbito productivo sino también en el organizacional por lo que se requieren de nuevas competencias que permitan no sólo la aplicación del conocimiento sino también su generación y así estar en posibilidades de realizar investigación e innovación en las empresas. Por tanto, el conocimiento se ha convertido en el elemento central del nuevo paradigma productivo, donde la transformación educativa pasa a ser un factor fundamental para el desarrollo de la innovación y la creatividad (Punie, 2007 y Salas, 2013). Así, la educación se ha convertido en el insumo principal cuya función es la formación del capital humano capacitado acorde a las necesidades impuestas por la globalización, así como la de propiciar conocimientos científicos y tecnológicos innovadores que permitan producir un valor agregado a la estructura económica de los países, entre los que se encuentran los programas de nivel posgrado. Desde esta perspectiva económica, se enfatiza la importancia no sólo del capital humano sino también la relacionada con la gestión del nuevo conocimiento, de la innovación y del desarrollo de las capacidades humanas como fuentes de crecimiento económico sostenible (Ananiadou y Claro, 2009). Para alcanzarlo, es mediante el desarrollo del sistema de educación superior que incentive la generación de capital humano con base en el diseño de ambientes de aprendizaje orientados hacia: a) una situación auténtica como fuente de especificación de las competencias; b) el estudiante como protagonista y agente de la acción; c) el fomento de la competencia, a la acción y a los recursos; d) la comunidad que propicia la colaboración con otros estudiantes y miembros de la misma; y e) la evaluación para realimentar en formación y para tener una valoración de su desempeño (McGaghie, Issenberg, Petrusa y Scalese, 2010).

De esta manera, surge la sociedad del conocimiento la cual ha motivado cambios al incorporar a la ciencia y tecnología como fuerzas productivas que permiten el desarrollo de los países por lo que éstos tienen que concentrarse en la generación de capacidades investigativas e innovativas en la educación superior (Gómez, Aranda y Santos, 2017 y Herrera y Didriksson, 1999). No obstante, para México, se presenta el siguiente diagnóstico sobre la generación de conocimiento e innovación con fundamento en los Indicadores de Ciencia y Tecnología elaborados por la OECD (2014): El número de investigadores por cada mil habitantes es de 0.98 (penúltimo lugar), por arriba de Chile con el 0.87, en donde el primer lugar fue Finlandia con el 15.88 para el año 2012; el porcentaje de patentes otorgadas por condición de residencia en 2012 para México fue del 8\% en comparación con Japón con el $85 \%$ y Estados Unidos con el $50.6 \%$; el número de artículos publicados por cada mil habitantes para el año 2012 en México fue 0.09 en comparación con Canadá con el 1.70 y Estados Unidos con el 1.14; el Gasto Interno Bruto en Investigación y Desarrollo como porcentaje del PIB para el año 2011 fue de 0.43 en comparación con Corea con el 4.04 y el Gasto en Educación Superior en Investigación y Desarrollo en 2011 fue de 0.12 en contraste con Dinamarca con el 0.94 .

Ante la situación anterior, México reconoce que la capacidad de innovación es un factor prioritario para el desarrollo del país en donde el posgrado tiene la responsabilidad de formar las competencias en los estudiantes que les permitan generar nuevo conocimiento tanto científico como tecnológico enfocado a satisfacer las necesidades y requerimientos de los sectores productivos y sociales, así como propuestas innovadoras que permitan mejorar los niveles de vida. En este sentido, el Consejo Nacional de Ciencia y Tecnología (CONACyT) en su Programa Especial de Ciencia, Tecnología e Innovación 2014 - 2018 (PECITI, 2014) estableció como uno de sus objetivos estratégicos el formar y fortalecer capital humano altamente calificado que posibilite la transición a una economía basada en el conocimiento. Así, es fundamental la generación de las competencias relacionadas con la investigación en el capital humano mediante el crecimiento de la oferta de posgrados, específicamente aquella relacionada con el Programa Nacional de Posgrados de Calidad (PNPC) del CONACyT con la finalidad de brindar opciones de calidad a los estudiantes que estén interesados por fortalecer su formación científica y tecnológica. 
De este modo, Cardoso y Cerecedo (2011) señalan que la calidad educativa del posgrado es sinónimo de producción de conocimiento por lo que se ha constituido en el principal factor que permite acreditar a un programa por lo que mencionan que es de calidad siempre y cuando los procesos formativos generen capacidades, habilidades y destrezas útiles que les permitan a los egresados diseñar e implementar estrategias de intervención que permitan resolver las problemáticas del sector laboral. Por consiguiente, el nivel educativo de posgrado se ha constituido en el lugar preponderante en donde los profesionales tienen la oportunidad de complementar su formación para ampliar sus oportunidades de desarrollo en las áreas de la docencia e investigación, así como profundizar en un campo del conocimiento específico, por lo que ofrece tres niveles de formación: especialización, maestría y doctorado por lo que este se ha constituido como el responsable de propiciar la generación de nuevos conocimientos mediante la investigación científica, tecnológica, humanística y social. De ahí que las personas que acceden a este nivel es fundamental que adquieran o fortalezcan un conjunto de competencias relacionadas con la investigación que les posibiliten la aplicación y generación de conocimiento científico y tecnológico en el sector productivo, social y de servicios (Gekara y Snell, 2018 y Carreño, 2011).

Asimismo, con fundamento en Valdés, Vera y Carlos (2012) la relevancia de evaluar las competencias investigativas es fundamental debido a la globalización económica, en donde la gestión del conocimiento científico y tecnológico es un elemento relevante para que un país se desarrolle en forma holística, por lo que el presente estudio brinda información trascendental para los posgrados en administración en cuanto a los perfiles de ingreso de los estudiantes para así estar en posibilidad de instrumentar e implementar acciones formativas orientadas a la consolidación de estas competencias a nivel posgrado.

\section{ANTECEDENTES SOBRE LAS COMPETENCIAS INVESTIGATIVAS}

Así, surge la relevancia de conceptualizar las competencias investigativas. Tobon (2008) considera que es un proceso complejo en el que se encuentran los componentes cognitivos, metacognitivos, cualidades de la personalidad, motivación, experiencia social y profesional del sujeto. Por su parte, Maldonado, Landazábal, Hernández, Ruíz, Claro, Vanegas y Cruz (2007) las definen como aquellas que se orientan a la formación de profesionales con amplios conocimientos y destrezas para emprender proyectos para la resolución de problemas de relevancia social, económica o política. Así, esta formación implica ordenar y sistematizar las acciones de las personas para propiciar la gestión del conocimiento, así como la toma de decisiones en forma argumentada. Del mismo modo, Sánchez y Tejeda (2010) establecieron que las competencias investigativas están constituidas en rasgos como el indagativo, argumentativo, innovador, tecnológico y conductual. En tanto, Pirela y Prieto (2006) así como Valdés, Vera, Nenninger y Haydee (2012) señalan que las competencias necesarias para la investigación se dividen en genéricas y técnicas. Las primeras están relacionadas con las habilidades, cualidades y de relaciones humanas; mientras que las segundas, se enfocan sobre el conocimiento y técnicas específicas del área de estudio. En tanto, Potolea (2013) considera que esta clase de competencias están constituidas por las relacionadas con el conocimiento, las de personalidad e instrumentales. Las primeras están orientadas hacia la explicación y análisis del objeto de estudio, así como al empleo adecuado del lenguaje científico. Las segundas, se enfocan en la reflexión constructiva, crítica y ética, ser creativo e innovativo en la investigación aunado con la responsabilidad y autonomía, mientras que las terceras se focalizan hacia la resolución de problemas y a la aplicación y transferencia de situaciones reales al campo de la investigación.

En lo que respecta a los estudios previos sobre las competencias investigativas se encuentra el realizado por Rubio, Torrado, Quirós y Valls (2016) en la formación del estudiantado de posgrado en el área de Pedagogía, los cuales encontraron que una autopercepción elevada de sus competencias investigativas destacando la escritura académica y en menor medida los aspectos metodológicos, específicamente los conocimientos sobre el enfoque cuantitativo referentes al diseño y aplicación de instrumentos de recogida de información tipo escalas y cuestionarios, así como el análisis de datos cuantitativos mediante la estadística. De igual modo, Hodgson, Varsavsky y Matthews (2014) evaluaron las percepciones de los estudiantes de ciencia en seis componentes: conocimiento científico, comunicación oral, escritura científica, habilidades cuantitativas, trabajo en equipo y pensamiento ético a medida que se acercan a su graduación. El resultado principal fue que estas competencias se desarrollan a partir de situaciones de aprendizaje en laboratorios y mediante el proceso de las tutorías realizadas por los académicos, así como recomiendan llevar a cabo una investigación amplia para medir realmente las competencias que los estudiantes adquieren en todo el programa educativo. Mientras que en el estudio realizado por Valdés, Vera y Carlos (2012) se encontró que los estudiantes de posgrado de las áreas de ciencias naturales e ingenierías, afirman que los desarrollos de sus competencias son favorecidos en la medida en que participan en proyectos de investigación, establecen vínculos con los investigadores aunado a que destinan un mayor tiempo para llevar a cabo dicho proceso. Asimismo, Aular, Marcano y Moronta (2009) hallaron dos competencias investigativas en proceso de consolidación y que fueron la de caracterizar los tipos de investigación con un dominio regular, así como la de diseñar un instrumento (cuestionario o entrevista). 
En tanto, Harrison, Ray Hernández, Cianelli, Rivera y Urrutia (2005) determinaron la existencia de un dominio bueno en los estudiantes de licenciatura en enfermería acerca de identificar situaciones de su entorno profesional, definir los objetivos del estudio y la de elegir un tipo de investigación. En tanto, Olehnovica, Bolgzda y Kravale (2015) clasificaron a las competencias investigativas en tres grupos: información, comunicación y metodológicas, hallando resultados tales como un dominio consolidado en la búsqueda y análisis de la información, así como en el uso de las TIC y base de datos; no obstante, un nivel de logro insuficiente en las relacionadas con las de comunicación y metodológicas por lo que las consideran como un factor de riesgo en la formación a nivel posgrado.

De esta forma, el empleo de las TIC se ha integrado como una competencia a desarrollar en el alumnado de posgrado lo cual se le ha llamado como digital y que hace referencia a la capacidad de acceder, entender, transformar, utilizar y compartir la información que se encuentra en la red, por lo que incluye la de su procesamiento para convertirla en conocimiento. Así, la competencia digital comprende las habilidades y actitudes para analizar, sintetizar y evaluar la relevancia y fiabilidad de la información en forma productiva (OECD, 2003). También, esta competencia ha sido considerada por la Comisión Europea (2007) como una de tipo clave para el aprendizaje permanente y que incluye una combinación de conocimientos, capacidades y actitudes enfocadas hacia el empleo seguro y reflexivo de las TIC en la sociedad del conocimiento; por lo que es relevante incluirla dentro de la formación de posgrado del alumnado debido a que gran proporción de ellos ya han crecido e interactuado en un entorno tecnológico en donde tienen que desarrollar un conjunto de competencias no sólo cognitivas sino también emocionales y sociales enfocadas hacia el acceso, la creación, utilización y comunicación con base en las TIC en forma creativa y analítica (Hobbs, 2010 y Palfrey y Gasser, 2008). Por consiguiente, fue importante realizar el diagnóstico de los estudiantes que iniciaban sus estudios de posgrado en administración para identificar aquellas competencias que manejan en investigación debido a que durante su trayecto formativo tienen que elaborar un proyecto terminal para así estar en posibilidades de obtener el grado correspondiente, así como desarrollar los elementos teóricos y metodológicos que les permitan utilizar estas competencias para la elaboración de estrategias de intervención en el sector productivo, lo cual les posibilitará intervenir ante las problemáticas y necesidades que se originan continuamente en el ámbito laboral.

También, como lo mencionan Thomas, Martin y Pleasants (2011) que la transformación hacia una sociedad del conocimiento constituye una oportunidad para las IES que, al enriquecer su misión y perspectivas asumirán un papel estratégico en la generación de conocimientos y en la formación de una masa crítica que posea mayor capacidad de propuesta para la construcción de esquemas sustentables de organización social. Por consiguiente, la educación posibilita a las personas agregar valor a la economía además de contribuir al patrimonio cultural, participar en la sociedad, mejorar la salud de sus familias y comunidades, preservar el medio ambiente e incrementar su propia capacidad para continuar desarrollándose. Asimismo, ante la evaluación realizada por el CONACyT a los programas de posgrado en donde uno de los elementos es la generación de productos de carácter científico y tecnológico por parte del estudiante, es necesario identificar sus perfiles de ingreso en cuanto a los niveles de dominio que poseen acerca de las competencias investigativas para así estar en posibilidades de lograr su consolidación. Lo anterior se confirma por lo realizado por Sánchez (2008), UNESCO (2009) y Valdés, Vera y Carlos (2012) quienes determinaron que el desarrollo de las competencias investigativas es un componente esencial de la formación del posgrado.

Por tanto, el objetivo general de la investigación fue valorar el nivel de desarrollo inicial de las competencias investigativas del estudiantado de los posgrados en administración tanto a nivel maestría como doctorado. Con base en la literatura previa, para este estudio se definieron a las competencias investigativas como el conjunto de habilidades, destrezas y actitudes que permiten elaborar y desarrollar proyectos a partir de la detección de problemáticas de relevancia en el sector de productos y servicios para analizarlas con el fin de diseñar las respectivas estrategias de solución que permitan generar un avance científico y tecnológico. Así, las dimensiones que integran a las competencias investigativas están orientadas hacia el diseño del estudio, el empleo de métodos y técnicas de investigación instrumental, el uso de estrategias cognitivas por parte del individuo y la difusión de los resultados.

\section{METODOLOGÍA}

Con base en Hernández, Fernández y Baptista (2011), se trató de una investigación no experimental cuantitativa con un alcance exploratorio-descriptivo, porque se enfocó en el diagnóstico de las competencias investigativas de los estudiantes que iniciaban su formación en el nivel de posgrado con campo en administración. Además, fue descriptiva debido a que se orientó en determinar el nivel de dominio de las competencias investigativas en cada uno de los programas de posgrado seleccionados para diagnosticas los perfiles de ingreso. 
La población objeto de estudio estuvo conformada por 185 estudiantes que comenzaban su trayecto formativo a nivel posgrado en seis programas orientados al campo en administración y que fueron: a) Maestría en Alta Dirección (MAD); b) Maestría en Administración (MA); c) Maestría en Administración y Políticas Públicas (MAAP); d) Maestría en Administración de Empresas Sustentables (MAES); e) Doctorado en Ciencias Administrativas (DCA) y f) Doctorado en Gestión y Políticas de la Innovación Organizacional (DGPI). Para determinar el tamaño óptimo de la muestra para una población finita se utilizó el procedimiento establecido por Martínez (2012) y Münch y Ángeles (2007), considerando las siguientes condiciones: Una población de 185 personas, una probabilidad de éxito de 0.5 (p), un nivel de confianza del 95\%, por lo que obtuvo una muestra representativa de 124 estudiantes distribuidos en los cinco programas de posgrado.

Para el diseño del instrumento se consideraron las aportaciones de Potolea (2013) y Valdés, Vera, Nenninger y Haydee (2012), por lo que se elaboró un cuestionario basado en una escala Likert y organizado del siguiente modo: 1) Datos sociodemográficos y 2) Evaluación inicial de las competencias investigativas con cuatro componentes: diseño, instrumentales, personales y gestión para la divulgación. Los ítems se midieron a través de una escala con cinco opciones de respuesta comprendida de 0 (no desarrollada) hasta 4 (muy desarrollada). La interpretación que se llevó a cabo fue a partir de los siguientes criterios: valores superiores de tres a cuatro significan dominios altos; valores mayores de dos a tres como dominios moderados; valores superiores de uno a dos como dominios insuficientes y el valor de cero a uno, como no desarrollada la competencia sujeta de evaluación.

El instrumento se le realizó un juicio de expertos para determinar la validez de su contenido para después obtener su fiabilidad y consistencia interna mediante el coeficiente alfa de Cronbach, la cual fue de 0.828 por lo que se interpreta como adecuada. Posteriormente, se efectuó la validez de constructo del instrumento con base en el análisis factorial exploratorio: La prueba estadística de KMO tuvo un resultado de 0.812 aunado al de la prueba de esfericidad de Barlett que fue estadísticamente significativo $(p=0.00)$, por lo que se determinó una buena adecuación de los datos a un modelo factorial. Así, se llevó a cabo el análisis de validez de constructos para los tres apartados del instrumento encontrándose que para la sección de las competencias investigativas sobre el diseño se hallaron cinco factores (autovalores iniciales mayores a uno) y que explican el $75.89 \%$ de la varianza total; mientras que para el apartado acerca de las instrumentales, se identificaron seis componentes (eigenvalue mayor que uno) que explican el 68.42\% de la varianza total. En tanto para el tercer apartado referente a las personales se obtuvieron cinco factores con una varianza de $72.55 \%$ y para la dimensión de la gestión para la divulgación, se hallaron cinco componentes que explican el $70.53 \%$ de la varianza total.

\section{RESULTADOS}

Los principales resultados de la investigación se presentan en subsecciones: (i) Hallazgos sociodemo-gráficos de los participantes; y (ii) Hallazgos sobre las competencias investigativas de los participantes.

\section{Hallazgos sociodemográficos de los participantes}

El 32\% del total correspondió al programa de MAD; el 17\% al programa de MA; el 15\% al programa de MAP; el $23 \%$ al programa de MAES; el $5 \%$ al programa de DCA y el $8 \%$ restante al programa de DGPI. Con respecto al género, la distribución muestral fue la siguiente: Para los programas de MAD, MA, MAES y DCA sobresale la participación de las mujeres con el 70\%, 67\%, 64\% y 67\%, respectivamente. Por el contrario, en los programas de MAP y DGPI, los hombres predominan con el $68 \%$ y $60 \%$. En cuanto a la edad de los participantes se halló que el $42 \%$ tiene de 30 a 34 años; el 33\% de 25 a 29 años; el $15 \%$ de 35 a 39 años y el $10 \%$ restante, de 40 a 44 años. La edad promedio fue de 32.1 años con una desviación típica de 4.7 años. Por su parte, la experiencia laboral de los participantes, se encontró una media de 8.8 años con una desviación típica de 4.6 años, sobresaliendo aquellos que tienen de 5 a 9 años trabajando con el $37 \%$. Esta información permite deducir que los encuestados poseen una trayectoria laboral después de haber concluido sus estudios de licenciatura.

Lo anterior se confirma por el hallazgo en lo que respecta al tamaño de la empresa en donde se encuentran laborando los participantes según la clasificación de empresas por número de trabajadores de la Secretaría de Economía (Legislativa, 2009): El 43\% se encuentra en empresas grandes con integrantes de 251 en adelante; el $36 \%$ se ubica en empresas medianas y el $21 \%$ restante, lo hace en pequeña y microempresa integrada por de 11 a 50 empleados. Del mismo modo, la actividad económica en la que se desarrolla la empresa en donde trabajan los participantes del estudio se distribuyó en un $58 \%$ en la industria manufacturera; el sector de servicios financieros con el $29 \%$; el $10 \%$ al turismo y el $3 \%$ restante, al sector de la educación. 
Asimismo, el motivo fundamental para realizar un posgrado en administración, los encuestados manifestaron en un $64 \%$ fue por consolidar su desempeño laboral; el $22 \%$ fue por ampliar sus oportunidades de trabajo y el $14 \%$ restante, para acceder a impartir docencia en el nivel superior. También se obtuvo información importante sobre su experiencia en la participación de proyectos de investigación tanto en sus estudios de licenciatura o en el sector laboral, obteniéndose que el $88 \%$ no posee ningún acercamiento y el $12 \%$ sí ha tenido como becarios.

\section{Hallazgos sobre las competencias investigativas de los participantes}

Para el primer componente de las competencias investigativas relacionadas con el diseño de investigación, se halló que el estudiantado al iniciar sus estudios de posgrado en administración en forma global posee un dominio insuficiente en lo que concierne a identificar situaciones de su entorno profesional susceptibles de ser investigadas, formular estratégicamente los objetivos o preguntas de estudio, establecer el planteamiento del problema y elaborar el marco de referencia. Sin embargo, se diagnosticó que en los programas de doctorado (DCA y DGPI), existen casos en donde el nivel de desarrollo es moderado con el $33 \%$ y $30 \%$, respectivamente; en tanto que el programa de MAD es el que tiene el nivel de desarrollo más bajo.

Para el segundo componente de las competencias investigativas de tipo instrumental, el alumnado posee un dominio insuficiente en lo relacionado con la determinación del tipo de estudio, diferenciar los tipos de muestreo, diseñar los instrumentos, organizar e interpretar los resultados, entre otros. No obstante, los programas de doctorado tienen un nivel de desarrollo moderado en comparación con los de maestría; mientras que los programas de MAD y MAES fueron los que tienen el menor nivel de desarrollo con el $68 \%$ y $65 \%$, respectivamente. Para el tercer componente orientado a las competencias personales en el ámbito de la investigación se diagnosticó que el estudiantado posee en su mayoría un nivel de desarrollo insuficiente, en donde los programas de maestría de MAD, MA y MAPP sobresalen con un $60 \%$ y $74 \%$. En tanto la MAES se encontró que un $57 \%$ se valoraron como no desarrollada. Por su parte, los programas de doctorado, el $33 \%$ del DCA y $40 \%$ del DGPI se percibieron con un nivel de dominio alto.

Para el cuarto componente sobre la gestión para la divulgación de los resultados de la investigación, se identificó que los participantes tienen un dominio no desarrollado en lo que respecta a la elaboración y publicación de productos académicos como ponencias en congresos, artículos en revistas internacionales 0 nacionales y formulación de capítulos de libros con un 95\% para el programa de MAD; 89\% para MAPP; 86\% para MA y $82 \%$ para MAES. De igual modo, el programa de DCA tiene un nivel no desarrollado con un $50 \%$; mientras que el de DGPI, sobresale con un dominio insuficiente que corresponde a un $50 \%$, un $30 \%$ para no desarrollado y solamente el $20 \%$ con un nivel moderado.

En lo referente a los niveles de dominios de las competencias investigativas con fundamento en la media y desviación estándar (s), se diagnosticó en términos a nivel posgrado (maestría y doctorado) que se presenta un nivel de desarrollo insuficiente para la gestión para la divulgación, diseño de investigación e instrumentales; mientras que con un dominio moderado en las de tipo personal (Tabla 1).

Tabla 1: Competencias investigativas del estudiantado en los posgrados en administración

\begin{tabular}{|l|c|c|c|c|c|c|c|c|}
\hline \multirow{2}{*}{ Programa } & \multicolumn{2}{|c|}{$\begin{array}{c}\text { Diseño de } \\
\text { investigación }\end{array}$} & \multicolumn{2}{c|}{ Instrumentales } & \multicolumn{2}{c|}{ Personales } & \multicolumn{2}{c|}{$\begin{array}{c}\text { Gestión para la } \\
\text { divulgación }\end{array}$} \\
\cline { 2 - 10 } & Media & $s$ & Media & $s$ & Media & $s$ & Media & $s$ \\
\hline MAD & 1.78 & 0.28 & 1.38 & 0.36 & 1.65 & 0.21 & 1.05 & 0.24 \\
\hline MA & 1.76 & 0.21 & 1.29 & 0.33 & 1.52 & 0.35 & 1.14 & 0.25 \\
\hline MAPP & 1.79 & 0.22 & 1.42 & 0.43 & 1.73 & 0.25 & 1.11 & 0.23 \\
\hline MAES & 1.17 & 0.20 & 1.43 & 0.36 & 1.46 & 0.39 & 1.18 & 0.21 \\
\hline DCA & 2.33 & 0.42 & 2.83 & 0.51 & 3.33 & 0.27 & 1.67 & 0.41 \\
\hline DGPI & 2.20 & 0.46 & 2.80 & 0.48 & 3.20 & 0.31 & 1.90 & 0.38 \\
\hline Total $(n=124)$ & 1.85 & 0.30 & 1.87 & 0.43 & 2.15 & 0.30 & 1.34 & 0.29 \\
\hline
\end{tabular}

En los resultados para cada uno de los programas de posgrado, la valoración fue la siguiente: Para el programa de MAD en los cuatro componentes se ubican con un nivel de dominio insuficiente en donde la media mayor corresponde al diseño de investigación; para el programa de MA también se halló un resultado similar; para el programa de MAPP se encontró en una situación similar y para el programa de MAES de igual forma, solo que sobresale en el componente de las competencias personales. Para los programas de DCA y DGPI, para el aspecto de la gestión para la divulgación se diagnosticó su media menor con un nivel insuficiente, mientras que la mayor le correspondió a las de tipo personal con un dominio moderado. 
Por el contrario, la competencia con mayor valoración por parte del alumnado correspondió a las de tipo personal y que se orientan hacia el desarrollo del pensamiento crítico, la reflexión, análisis y síntesis que permitan la organización de los hallazgos con una media de 2.15. Posteriormente, las competencias instrumentales obtuvieron el promedio alto con una media de 1.87 en donde hacen referencia a la formación sobre las técnicas y métodos de investigación con la formulación de los instrumentos de obtención de información. Para este resultado, se identificó que los programas de doctorado obtuvieron el puntaje más alto. En la siguiente fase del análisis de resultados se empleó la estadística inferencial con la finalidad de determinar la existencia en términos estadísticos de diferencias entre la formación del estudiantado en las competencias investigativas, por lo que se emplearon tanto la prueba "t" de Student como ANOVA para grupos independientes. Para comprobar el contraste entre grupos se utilizó la prueba post-hoc de Scheffé.

Para la variable género, no se hallaron diferencias entre hombres y mujeres en lo que respecta a sus competencias investigativas $(t=1.035, p>0.05)$. Este hallazgo significa que el estudiantado percibe del mismo modo su nivel de dominio de las competencias investigativas. De la misma manera, se hallaron diferencias en cuanto al tipo de régimen (público y privado) para las competencias investigativas $(t=4.249,<$ 0.05). Este hallazgo implica que el alumnado que cursa su posgrado en una institución pública posee un dominio diferente con aquellos que lo hacen en una institución privada. Se encontraron diferencias entre los programas educativos evaluados en cuanto a las competencias investigativas del alumnado (ANOVA, F $=18.55, p<0.05)$. Este resultado permite deducir la existencia de un perfil distinto para aquellos estudiantes que inician su maestría con respecto a los de doctorado. Asimismo, se consideró a la edad como una variable del estudio, en donde se determinaron diferencias del primer grupo (25 a 29 años) con respecto a los otros tres en las competencias investigativas (ANOVA, $F=21.54, p<0.05$ ). También, se halló una diferencia con respecto a la variable experiencia laboral del segundo grupo (5 a 9 años) con relación a los otros tres en lo concerniente a las competencias investigativas (ANOVA, $F=18.33, p<0.05$ ).

De esta forma, los hallazgos del estudio se relacionan con lo que afirman Valdés, Vera, Nenninger y Haydee (2012), los cuales establecieron que es preponderante determinar si los posgrados están cumpliendo con su función de formación del capital humano, por lo que estos resultados proporcionan un panorama inicial sobre lo que se requiere desarrollar en el alumnado en el campo de la administración para así estar en posibilidades no sólo de diseñar y desarrollar proyectos de investigación, sino también lograr una gestión eficiente y eficaz para la divulgación de los resultados a nivel nacional e internacional. Asimismo, se encontró un resultado similar con el realizado por Rubio, Torrado, Quirós y Valls (2016) en lo referente a la valoración baja de las competencias investigativas en el ámbito instrumental relacionado con el diseño y aplicación de instrumentos para obtener información y su organización respectiva. Por otro lado, los resultados del estudio son distintos con los hallazgos de Aular, Marcano y Moronta (2009) en lo referente al nivel moderado de dominio de las competencias relacionadas con el diseño de la investigación. También, es importante mencionar la existencia de diferencias en las competencias investigativas para las variables programa educativo, tipo de régimen, edad y experiencia laboral; mientras que para la de género, no se presentaron desde la perspectiva de los encuestados.

Por último, los resultados obtenidos en este estudio aportan una visión particular sobre las competencias investigativas en el nivel de posgrado con campo en administración permitiendo identificar las condiciones con las que ingresan los estudiantes a estos programas, por lo que se sugiere llevar a cabo un seguimiento sobre el desarrollo de dichas competencias en cuanto a su participación en los proyectos de investigación, el tiempo dedicado a realizarla, realización de estancias, así como la elaboración de los productos académicos relacionados con la divulgación del conocimiento. Esta recomendación coincide con el estudio realizado por Hodgson, Varsavsky y Matthews (2014) y confirma lo señalado por Adalid (2011) que de los diferentes niveles en que se divide la enseñanza en México, el posgrado tiene el propósito de formar individuos que participen en la investigación básica y aplicada orientada a la innovación, a crear tecnología aunado a difundir y transmitir el avance del conocimiento, así como lo establecido por Mercado, Cernas y Nava (2016), en lo que se refiere a la formación de individuos con una calidad educativa que sean impulsores del avance científico, tecnológico y humanístico capaces de generar conocimiento que apoye la toma de decisiones a los problemas y necesidades del sector laboral.

\section{CONCLUSIONES}

Los resultados encontrados en este trabajo de investigación permiten obtener las siguientes conclusiones: 1) Se logró el objetivo general porque se valoró el nivel de desarrollo inicial de las competencias investigativas del estudiantado de los posgrados en administración tanto a nivel maestría como doctorado, obteniéndose información valiosa acerca de los niveles de dominio en tres factores: diseño, desarrollo y gestión para la divulgación; 2) Los estudiantes se valoraron con un dominio insuficiente en sus competencias investigativas con base en el promedio para los componentes de diseño, instrumentales y gestión para la divulgación en los 
seis programas por lo que se aprecia una autovaloración deficitaria del alumnado lo que implica que consideran mínimas sus habilidades y destrezas relacionadas con la formulación y desarrollo de investigaciones científicas. Solamente se diagnosticó un dominio moderado para las competencias de tipo personal, 3) Los hallazgos obtenidos permiten elaborar un diagnóstico sobre los perfiles de ingreso del alumnado que inicia su formación en los posgrados del área de administración y, 4) Se sugiere que se lleven a cabo otros estudios que permitan corroborar o refutar los datos obtenidos en la presente investigación sobre todo en los posgrados del área de ingeniería, ciencias de la salud y educación.

\section{AGRADECIMIENTOS}

Los autores agradecen al Instituto Politécnico Nacional las facilidades otorgadas para la realización de este estudio a partir de la autorización de los proyectos de investigación: SIP20181014 titulado "Valoración del capital intelectual en los posgrados en ciencias sociales y administrativas" y SIP20170079 titulado "El proceso de gestión de la educación superior y su incidencia en la gobernabilidad".

\section{REFERENCIAS}

Adalid, C., CONACyT y el Posgrado: Políticas de Evaluación y Calidad, Gestión y Estrategia, 40, 87 - 98 (2011)

Akareem, H. y S. Hossain, Perception of Education Quality in Private Universities of Bangladesh: A study from Students' Perspective, doi: 10.1080/08841241.2012.705792, Journal of Marketing for Higher Education, 22(1), 11-33 (2012)

Ananiadou, K. y M. Claro, 21ST Century Skills and Competences for New Millennium Learners in OECD Countries, doi: 10.1787/19939019, OECD Education Working Papers, 41, 1-34 (2009)

Aular de Durán, J., N. Marcano y M. Moronta, Competencias Investigativas del Docente de Educación Básica, https://goo.gl/3YbuoJ, Laurus, 15(30), 138-165 (2009)

Brennan, J. y U. Teichler, The Future of Higher Education and of Higher Education Research, doi:10.1007/s10734-0089124-6, Higher Education, 56(3), 259-264 (2008)

Cardoso, E. y M. Cerecedo, Propuesta de Indicadores para Evaluar la Calidad de un Programa de Posgrado en Educación, https://goo.gl/dDqGVz, Revista Electrónica de Investigación Educativa, 13(2), 68 - 82 (2011)

Carneiro, R. y A. Draxler, Education for the 21st Century: Lessons and Challenges, doi:10.1111/j.14653435.2008.00348.x/pdf, European Journal of Education, 43 (2), 149-160 (2008)

Comisión Europea, Competencias Clave para el Aprendizaje Permanente. Un Marco de Referencia Europeo. Oficina de Publicaciones Oficiales de las Comunidades Europeas, Luxemburgo (2007)

Delamare, F. y J. Winterton, What is Competence? Human Resource Development International, 8 (1), 27 - 46 (2007)

Gekara, V. y D. Snell, Designing and Delivering Skills Transferability and Employment Mobility: The Challenges of a Markent-Driven Vocational Education and Training System, J. Vocational Education \& Training, 70 (1), 107 - 129 (2018)

Gobierno de la República, Programa Especial de Ciencia, Tecnología e Innovación 2014 - 2018, Gobierno de la República, México (2014)

Gómez, M., E. Aranda y J. Santos, A Competency Model for Higher Education: An Assessment Based on Placements, Studies in Higher Education, 42 (12), 2195 - 2215 (2017)

Harrison, L., A. Ray Hernández, R. Cianelli, M. Rivera y M. Urrutia, Competencias en Investigación para Diferentes Niveles de Formación de Enfermeras: Una Perspectiva Latinoamericana, https://goo.gl/BfC8Mz, Ciencia y enfermería, 11(1), $59-71$ (2005)

Hernández, R., C. Fernández y P. Baptista, Metodología de la Investigación, Mc Graw Hill, México (2011)

Herrera, A. y A. Didriksson, La Construcción Curricular: Innovación, Flexibilidad y Competencias, Educación Superior y Sociedad, 10(2), 29 - 52 (1999)

Hobbs, R., Digital and Media Literacy: A Plan of Action, The Aspen Institute, Washington, D.C. (2010)

Hodgson, Y., C. Varsavsky y K. Matthews, Assessment and Teaching of Science Skills: Whole of Programme Perceptions of Graduating Students, https://goo.gl/jGV23e, Assessment and Evaluation in Higher Education, 39 (5), 515 - 530 (2014)

Legislativa, A., Ley para el Desarrollo para la Competitividad de la Micro, Pequeña y Mediana Empresa del Distrito Federal, Asamblea Legislativa, México (2009)

Maldonado, L., D. Landazábal y otros cinco autores, Visibilidad y Formación en Investigación, Estrategias para el Desarrollo de Competencias Investigativas, https://goo.gl/1MoUZY, Studiositas, 2(2), 43 - 56 (2007)

Martínez, C., Estadística y Muestreo, Ecoe Ediciones, Bogotá, Colombia (2012) 
McGaghie, W., S. Issenberg, E. Petrusa y R. Scalese, A Critical Review of Simulation-Based Medical Education Research: 2003- 2009, doi:10.1111/j.1365-2923.2009.03547.x, Medical Education, 44, 50 - 63 (2010)

Mercado, P., D. Cernas y R. Nava, La Interdisciplinariedad Económico-Administrativa en la Conformación de una Comunidad Científica y la Formación de Investigadores, https://goo.gl/h9nqCi, Revista de la Educación Superior, XLV (177), $43-65$ (2016)

Münch, L. y E. Ángeles, Métodos y Técnicas de Investigación, Trillas, México (2007)

OECD, Los Desafíos de las Tecnologías de la Información y las Comunicaciones en la Educación, doi:10.1787/9789264103429-es, Madrid, España (2003)

OECD, Main Science and Technology Indicators, https://goo.gl/NnoGZm, OECD, París, Francia (2014)

Olehnovica, E., I. Bolgzda y M. Kravale, Individual Potential of Doctoral Students: Structure of Research Competences and Self-assessment, Procedia - Social and Behavioral Sciences, 174, 3557 - 3564 (2011)

Palfrey, J. y U. Gasser, Born Digital: Understanding the First Generation of Digital Natives, New York (2008)

Pirela, L. y L. Prieto, Perfil de Competencias del Docente en la Función de Investigador y su Relación con la Producción Intelectual, Opción, 22 (50), 159 - 177 (2006)

Potolea, D., Doctoral Studies and Research Competences, Procedia - Social and Behavioral Sciences, 76, 935 - 946 (2013)

Punie, Y., Learning Spaces: An ICT- Enabled Model of Future Learning in the Knowledge - Based Society, doi:10.1111/j.1465-3435.2007.00302.x/, European Journal of Education, 42 (2), 185 - 199 (2007)

Rubio, M., M. Torrado, C. Quirós y R. Valls, Autopercepción de las Competencias Investigativas en Estudiantes de Último Curso de Pedagogía de la Universidad de Barcelona para Desarrollar su Trabajo de Fin de Grado, Revista Complutense de Educación, 29 (2), 335 - 354 (2016)

Salas, S., Evolution of the Framework for 21st Century Competencies, https://goo.gl/11uS7i, Knowledge Management \& E-Learning: An International Journal, 5 (1), 10 - 24 (2013)

Sánchez, L., Proceso de Formación del Investigador en el Área Tecnológica, El Caso de los Programas de Posgrado del CENIDET, Revista de Educación Superior, XXXVII (145), 7 - 23 (2008)

Sánchez, P. y R. Tejeda, El Proceso de Formación Investigativa del Profesional Ingeniero y la(s) Competencia(s) Investigativa(s), https://goo.gl/9cS8ko, Revista Pedagogía Universitaria, XV (4), 37 - 47 (2010)

Thomas, G., D. Martin y K. Pleasants, Usign Self- and Peer- Assessment to Enhance Students' Future Learning in Higher Education. Journal of University Teaching and Learning Practice, 8 (1), 1 - 17 (2011)

Tobon, S., La Formación Basada en Competencias en la Educación Superior. El Enfoque Complejo. Magisterio, Bogotá, Colombia (2008)

UNESCO, Conferencia Mundial sobre la Educación Superior, 2009, La Nueva Dinámica de la Educación Superior y la Investigación para el Cambio Social y el Desarrollo, UNESCO, París, Francia (2009)

Valdés, Á., J. Vera y E. Carlos, Competencias Científicas en Estudiantes de Posgrado de Ciencias Naturales e Ingenierías, Sinéctica, https://goo.gl/27rG3d, 39, 1 - 17 (2012)

Valdés, Á., J. Vera, E. Nenninger y E. Haydee, Variables Asociadas al Desarrollo de la Competencia Científica en Estudiantes de Posgrado en Sonora, https://goo.gl/Mr9RvT, Reencuentro, 63, 40 - 46 (2012) 
